\title{
Prognostic Significance of Biomarkers in Predicting in-Hospital All-Cause Mortality in Elderly Patients with Acute Myocardial Infarction
}

\author{
M Broslavskyte ${ }^{1}$, R Serpytis ${ }^{2}$, G Navickas ${ }^{2}$, S Glaveckaite ${ }^{2}$, R Samalavicius ${ }^{3}$ and P Serpytis ${ }^{2}$ \\ ${ }^{1}$ Faculty of Medicine, Vilnius University, Vilnius, Lithuania \\ ${ }^{2}$ Clinic of Cardiovascular diseases, Institute of Clinical Medicine, Vilnius University Faculty of Medicine, Vilnius, Lithuania \\ ${ }^{3}$ Clinic of Emergency Medicine, Institute of Clinical Medicine, Vilnius University Faculty of Medicine, Vilnius, Lithuania \\ *Corresponding author: Margarita Broslavskytė, Faculty of Medicine, Vilnius University, Universiteto St, LT 01513 Vilnius, \\ Lithuania
}

\section{ARTICLE INFO}

Received: 幽 July 31, 2019

Published: 慧 August 13, 2019

Citation: Broslavskyte M, Serpytis R, Navickas G, Glaveckaite S, Samalavicius R, Serpytis P. Prognostic Significance of Biomarkers in Predicting in-Hospital All-Cause Mortality in Elderly Patients with Acute Myocardial Infarction. Biomed J Sci \& Tech Res 20(3)-2019. BJSTR. MS.ID.003462.

Keywords: Acute Myocardial Infarction; All-Cause Hospital Mortality; Biomarkers; Prognosis

Abbreviations: BNP: B-Type Natriuretic Peptide; HS-CRP: High-Sensitivity C-Reactive Protein; OR: Odds Ratio; STEMI: ST-Segment Elevation Myocardial Infarction

\section{ABSTRACT}

Background: AMI is a common cause of death in elderly patients. Therefore, prognostic prediction has become crucially important part of the treatment process.

Aim: We aimed to investigate prognostic significance of biomarkers and other clinical factors in predicting all-cause in-hospital mortality in patients older than 75 years with AMI.

Materials and Methods: 2059 consecutive patients were retrospectively included in single center study. Participants were divided into groups based on their in-hospital mortality. The prognostic ability of biomarkers peak values was evaluated by using ROC curve and binary logistic regression analysis.

Results: Among 2059 patients enrolled in this study, 1141 (55.4\%) were woman, and 1060 (51.5\%) were with a diagnosis of non-ST segment elevation myocardial infarction. The mean age (SD) of the study population was 81.97 (4.33) years. In-hospital mortality rate in our study was $13.3 \%$. Peak Troponin I, BNP and hs-CRP concentrations were significantly higher in deceased patients (all $\mathrm{p}<0.01$ ). The area under the ROC curve for Troponin I was 0.595, 0.653 for BNP and 0.664 for hs-CRP. BNP level $>824.3 \mathrm{ng} / \mathrm{l}$ and hs-CRP level $>78.7$ $\mathrm{g} / \mathrm{l}$ were disclosed as the best thresholds for mortality prediction in this age group. Using binary logistic regression, hs-CRP level >78.7 g/l (OR (95\% CI), 2.68 (1.89-3.81)), stroke history (OR (95\%CI), 2.3 (1.53-3.47)), BNP level >824.3 ng/l (OR (95\% CI), 2.04 (1.43$2.91)$ ), in-hospital bleeding complications (OR (95\% CI), 2.04 (1.27-3.28)) were identified as strongest independent predictors of in-hospital all-cause mortality.

Conclusion: In-hospital mortality in elderly patients with acute myocardial infarction is $13.3 \%$. Troponin I is the least useful biomarker in predicting mortality. Increased levels of hs-CRP, BNP, stroke history and the presence of any in-hospital bleeding complications were identified as reliable predictors of in-hospital mortality in the elderly population with acute myocardial infarction.

\section{Introduction}

Due to the fast growth of aged population, acute coronary syndrome is a major health problem worldwide. Elderly patients represent a large group of patients admitted to hospital with a diagnosis of AMI. Advanced age is an important risk factor of adverse outcomes of both invasive and conservative treatment methods [1,2]. Many elderly patients also suffer from other diseases that have short-term and long-term impact on their health.
The most common comorbidities are diabetes mellitus and chronic kidney disease $[3,4]$. While making decisions on the best treatment options, it is very important to evaluate each patient's prognosis and risk of complications or death. Although it is important to have a risk stratification tool suitable for this age group, elder population is often underrepresented in many clinical trials [5].Biomarkers used in myocardial infarction and heart failure diagnosis such as 
B-type natriuretic peptide, cardiac Troponin I and high-sensitivity $\mathrm{C}$ reactive protein can add a prognostic value while making a clinical evaluation [6-8]. The aim of this study was to evaluate the association between different diagnostic biomarkers and inhospital mortality in elderly patients with AMI.

\section{Material and Methods}

A retrospective single-center study of 2059 consecutively collected patients older than 75 years admitted with acute STsegment elevation myocardial infarction (STEMI) or non-ST segment elevation myocardial infarction (NSTEMI) was conducted. Patients were hospitalized in Vilnius University Hospital Santaros Clinics in Vilnius, Lithuania, between $1^{\text {st }}$ January 2012 and December $31^{\text {st }}, 2016$. AMI was diagnosed according to the $3^{\text {rd }}$ Universal Definition of Myocardial Infarction [9]. We collected the following data: sex, age, comorbidities such as atrial fibrillation, diabetes mellitus, hypertension, chronic kidney disease, history of previous myocardial infarction or ischemic stroke, Killip class on admission, highest Troponin I, BNP, hsCRP values, coronary angiography findings, treatment choices and all-cause mortality rate. In hospital bleeding complications were categorized into major and minor. Major bleeding was defined as bleeding associated with death, transfusion of more than 2 units of packed RBCs or whole blood, a reduction in the level of $\mathrm{Hb}>2 \mathrm{~g} / \mathrm{dL}$ or a serious lifethreatening clinical event requiring medical intervention. Minor bleeding event was considered as every bleeding event that does not fulfill the criteria of major bleeding. Clinical and demographic data were retrospectively evaluated from patients' medical data. All participants were categorized according to their treatment outcomes into deceased and alive patients' groups. Ethical approval for the study was obtained from local bioethical committee (Nr.158200-18/4-1015-522).

\section{Statistical Analysis}

Data analysis was performed using IBM SPSS 22 and MedCalc (version 18.11.3) statistical software. The normality of the data was checked by using the Shapiro-Wilk test. Continuous variables were expressed as mean and standard deviation (SD) or medians and interquartile range (IQR, Q1-Q3). Categorical variables were expressed as frequencies and percentages. Continuous variables were analyzed by using Mann-Whitney $U$ test and categorical variables were analyzed by using Chi-square test. Binary logistic regression was used to identify independent predictors of all-cause mortality of study population. The receiver operating characteristic (ROC) curve and area under the curve (AUC) with 95\% confidence index (CI) were calculated for Troponin I, BNP and hs-CRP. Cut off values, sensitivity and specificity for biomarkers to predict in-hospital mortality were calculated. All $\mathrm{p}$ values $<0.05$ were considered as statistically significant.

\section{Results}

Out of 2059 patients, 1060 (51.5\%) had a final diagnosis of NSTEMI and 999 (48.5\%) had a STEMI. 1141 (55.4\%) participants were women and 918 (44.6\%) were men. The mean (SD) age of the study population was 81.97 (4.33) years. 274 (13.3\%) patients have died during the hospital stay. The clinical characteristics of study patients in respect of in-hospital death are shown in Table 1. Some statistically significant differences were found between groups: older age, STEMI diagnosis, Killip class 4 and lower left ventricular ejection fraction (LVEF) on admission, presence of atrial fibrillation during the hospital stay, previous stroke history, conservative treatment approach, minor or major bleeding complications during in-hospital course were more common among patients that deceased. We found out that patients, that died, had significantly higher peak values of Troponin I (24415.97 vs. $11404.52 \mathrm{ng} / \mathrm{l}, \mathrm{p}<0.01$ ), BNP (1823.57 vs. $945.79 \mathrm{ng} / \mathrm{l}, \mathrm{p}<0.01$ ), CRP (107.95 vs. $51.54 \mathrm{~g} / \mathrm{l}, \mathrm{p}<0.01$ ) (Figure 1). ROC analysis was performed to determine the accuracy of these biomarkers and in order to find the best value that can predict patient's death. The AUC for Troponin I was 0.595 (Standard error (SE), 0.02; 95\% Confidence interval (CI), 0.57 to $0.62, \mathrm{p}<0.01$ ), 0.65 (SE, 0.02; $95 \% \mathrm{CI}, 0.63$ to $0.68, \mathrm{p}<0.01$ ) for BNP and 0.664 (SE, 0.02; $95 \% \mathrm{CI}$, 0.64 to $0.69, \mathrm{p}<0.01$ ) for hs-CRP. A cut-off value of BNP was 824.3 ng/l (sensitivity, 64.2\%; specificity, 62\%) and $78.7 \mathrm{~g} / \mathrm{l}$ (sensitivity, $50.64 \%$; specificity, $76.7 \%$ ) for hs-CRP. ROC comparison revealed no significant difference between BNP and hs-CRP $(\mathrm{p}=0.41)$ or Troponin I ( $p=0.07)$ AUCs. A weak significant $(p=0.01)$ difference was between Troponin I and hs-CRP AUCs. The mortality rate was higher among patients with BNP level $>824.3 \mathrm{ng} / \mathrm{l}$ (19\% vs. 7.5\%, $\mathrm{p}<0.01)$, hs-CRP level $>78.7 \mathrm{~g} / \mathrm{l}(19.4 \%$ vs. $8 \%, \mathrm{p}<0.01)$. Using binary logistic regression, we estimated that patients with hs-CRP levels $>78.7 \mathrm{~g} / \mathrm{l}$ were almost 3 times more likely to die during the hospital stay after AMI (OR (95\% CI), 2.68 (1.89-3.81), $\mathrm{p}<0.01$ ) and more than 2 times with BNP levels $>824.3 \mathrm{ng} / \mathrm{l}$ (OR (95\% CI), 2.04 (1.43-2.91), $\mathrm{p}<0.01)$. Patients, that had stroke history or that had a by bleeding complications during the stay in the hospital, were also more than 2 times likely to not survive (Table 2).

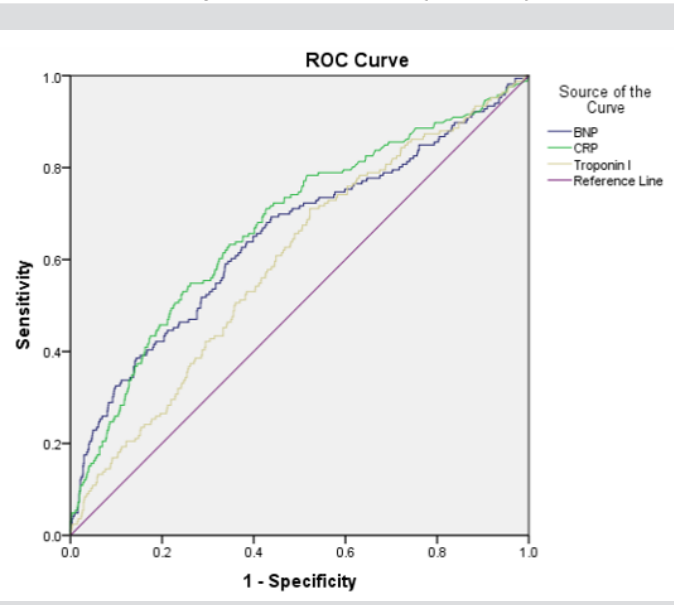

Figure 1: Troponin I, BNP and hs-CRP ROC curves for inhospital mortality prediction.

Abbreviations: AUC: Area Under the Curve; BNP: B-Type Natriuretic Peptide; HS-CRP: High sensitivity C Reactive Protein; ROC: Receiver Operating Characteristic Curve. 
Table 1: Characteristics of study population based on their survival.

\begin{tabular}{|c|c|c|c|}
\hline Characteristic & $\begin{array}{l}\text { Patient Survived } \\
\quad(n=1785)\end{array}$ & $\begin{array}{l}\text { Patient Died } \\
(n=274)\end{array}$ & $P$ value \\
\hline Age, years, mean (SD) & $81.76(4.26)$ & $83.35(4.54)$ & $<0.01$ \\
\hline Male, n (\%) & $804(45 \%)$ & $114(41.6 \%)$ & 0,29 \\
\hline Female, n (\%) & $981(55 \%)$ & $160(58.4 \%)$ & \\
\hline STEMI, n (\%) & $832(46.6 \%)$ & $167(60.9 \%)$ & $<0.01$ \\
\hline NSTEMI, n (\%) & $952(53.3 \%)$ & 107 (39.1\%) & \\
\hline Coronary angiography, n (\%) & $1624(91 \%)$ & $198(72.3 \%)$ & $<0.01$ \\
\hline PCI, n (\%) & $1310(73.4 \%)$ & $164(59.9 \%)$ & $<0.01$ \\
\hline Successful coronary stenting, n (\%) & $1254(70.3 \%)$ & $143(52.2 \%)$ & $<0.01$ \\
\hline CABG, n (\%) & $64(3.6 \%)$ & $10(3.6 \%)$ & 0.96 \\
\hline \multicolumn{4}{|c|}{ Killip class, n=1736 } \\
\hline Killip class 1, n (\%) & $791(53.4 \%)$ & $20(7.9 \%)$ & $<0.01$ \\
\hline Killip class 2, n (\%) & $464(31.3 \%)$ & $23(9.1 \%)$ & $<0.01$ \\
\hline Killip class 3, n (\%) & $93(6.3 \%)$ & $12(4.7 \%)$ & 0.32 \\
\hline Killip class 4, n (\%) & $134(9 \%)$ & $199(78.3 \%)$ & $<0.01$ \\
\hline $\begin{array}{l}\text { Ischemic or hemorrhagic } \\
\text { stroke history, n (\%) }\end{array}$ & $228(12.8 \%)$ & $59(21.5 \%)$ & $<0.01$ \\
\hline Previous MI, n (\%) & $467(26.2 \%)$ & $64(23.4 \%)$ & 0.32 \\
\hline Atrial fibrillation, n (\%) & $473(26.5 \%)$ & $107(39.1 \%)$ & $<0.01$ \\
\hline Diabetes mellitus, n (\%) & $341(19.1 \%)$ & $60(21.9 \%)$ & 0.52 \\
\hline LVEF, median (IQR), (\%) & $40 \%(15)$ & $30 \%(15)$ & $<0.01$ \\
\hline $\begin{array}{l}\text { Peak Troponin I, mean } \\
\text { (SD) ng/l }\end{array}$ & $11404.52(45737.82)$ & 24415.97 (80183.27) & $<0.01$ \\
\hline $\begin{array}{l}\text { Peak BNP, mean (SD) } \\
\text { ng/l }\end{array}$ & $945.79(1068.02)$ & $1823.57(2024.95)$ & $<0.01$ \\
\hline $\begin{array}{c}\text { Peak hs-CRP, mean (SD) } \\
\text { g/l }\end{array}$ & $51.54(71.01)$ & $107.95(106.54)$ & $<0.01$ \\
\hline Length of stay, median (IQR) days & $6(8)$ & $1(4)$ & $<0.01$ \\
\hline Bleeding (all cases), n (\%) & $133(7.5 \%)$ & $60(21.9 \%)$ & $<0.01$ \\
\hline Major bleeding, n (\%) & $34(1.9 \%)$ & $41(15 \%)$ & 0.32 \\
\hline Minor bleeding, $\mathrm{n}(\%)$ & $98(5.5 \%)$ & $19(6.9 \%)$ & $<0.01$ \\
\hline
\end{tabular}

Myocardial Infarction; NSTEMI: Non-ST Segment Elevation Myocardial Infarction; PCI: Percutaneous Coronary Intervention; SD: Standard Deviation; STEMI: ST-Segment Elevation Myocardial Infarction

Table 2: Binary logistic regression for predicting all-cause in-hospital mortality in elderly Patients.

\begin{tabular}{|c|c|c|}
\hline Variables & OR (95\% CI) & P value \\
\hline hs-CRP $>$ 78.7 g/l & $2.68(1.89-3.81)$ & $<0.01$ \\
\hline Stroke history & $2.3(1.53-3.47)$ & $<0.01$ \\
\hline BNP $>824.3 \mathrm{ng} / \mathrm{l}$ & $2.04(1.43-2.91)$ & 0.01 \\
\hline Bleeding & $2.04(1.27-3.28)$ & 0.03 \\
\hline Atrial fibrillation & $1.65(1.16-2.34)$ & 0.05 \\
\hline
\end{tabular}

Abbreviations: BNP: B-Type Natriuretic Peptide; HS-CRP: High-Sensitivity C-Reactive Protein; OR: Odds Ratio; STEMI: ST-Segment Elevation Myocardial Infarction.

\section{Discussion}

Many studies are still trying to find best biomarkers that might give an accurate mortality prediction, especially in elderly patients $[10,11]$. Although, many novel biomarkers are being tested, cardiac Troponin I, BNP and hs-CRP are widely used in the management of AMI $[12,13]$. It is important to evaluate their prognostic value. Studies, that tried to establish the prognostic role of these biomarkers in patients with cardiovascular diseases, found high accuracy of Troponin I (AUC 0.8) and N-terminal pro-brain natriuretic peptide (AUC 0.87) [14,15]. 
Sensitivity and specificity of biomarkers were also high in accessing the risk of death in common comorbidities or complications in elderly patients. These markers are valuable in predicting cardiovascular death in acute heart failure and might be useful in common comorbidities in elderly patients such as community-acquired pneumonia, severe sepsis or septic shock [16-18]. This work is one in a few studies focusing on risk assessing only in elderly and very elderly patients during AMI. Our study showed a significant association between selected biomarkers and cardiac mortality during the hospital stay, which was similar to other authors' results [19. 21]. Levels of all biomarkers were higher in non-surviving patients after AMI. Although, in our results specificity and sensitivity were lower than in earlier mentioned studies, it is important to note that our study consists only of elderly patients with many comorbidities and history of cardiovascular disease (CVD). In our study high-sensitivity Troponin I had a weakest predictive value in comparison to BNP or hs-CRP.

A prospective multicenter study by Gimenez et al. concluded that Troponin I show a high accuracy in early diagnosing myocardial infarction, but it does not have a good prognostic value as in our findings [22]. It is important to highlight that BNP level $>824.3$ $\mathrm{ng} / \mathrm{l}$ was found as a cut-off value to predict mortality in this age group. In similar studies lower BNP cut-off values were identified as a significant predictor of major adverse cardiovascular events (MACE), including death. However, patients in these studies had lower mean or patients aged more than 80 years old were as exclusion criteria and that might contribute to such results $[23,24]$ In contrast, studies with only older patients as participants showed much higher cut-off values as a mortality indicator $[25,26]$. These findings are important as some studies note that BNP loses its diagnostic power in elderly patients, but our results show that it can be a valuable biomarker for risk stratification $[27,28]$. Our analysis revealed that hs-CRP has the highest AUC with a specificity of $76.7 \%$ in predicting adverse outcomes. This biomarker plays a significant role in cardiovascular disease pathogenesis and in other conditions that contribute to all-cause mortality [29].

A recent study by Mani et al. revealed that initial and subsequent hs-CRP increase after 16 days was a good prognostic tool in predicting MACE, cardiovascular death and all-cause death. Patients with AMI and elevated hs-CRP concentration are at greater risk of inhospital death and are in need of close monitoring in order to avoid death or complications $[8,30]$. Furthermore, our research indicates that patients with elevated BNP and hs-CRP levels were three times more likely to die after AMI. These findings could be beneficial in better understanding of the elevated biomarkers impact during AMI within the elderly population. Knowing that greater BNP and hs-CRP levels lead to poor outcomes in this age group could increase our attention towards more effective management of these patients and treatment strategy decision. There are possible limitations of our research. One of them is retrospective nature of our study. Secondly, we did not evaluate long-term outcomes of study patients and prognostic abilities of biomarkers in follow-up. In addition, quality of life was not measured following myocardial infarction due to no data available on this topic.

\section{Conclusion}

In our study in-hospital mortality in elderly patients with acute myocardial infarction was $13.3 \%$. Hs-CRP and BNP elevation are important predictors of in-hospital mortality and should be evaluated in elderly patients during AMI. In contrast, Troponin I elevation is less accurate biomarker in risk stratification of inhospital all-cause mortality. Stroke in patients' history and inhospital bleeding complications are also relevant factors that have a prognostic value.

\section{Conflict of interest}

None declared.

\section{References}

1. Aguiar Rosa SA, Timóteo AT, Nogueira MA, Belo A, Ferreira RC (2017) Acute coronary syndrome in elderly- What is the place for invasive strategy? Eur Geriatr Med 8(1): 90-95.

2. Zijlstra F, De Boer MJ (2005) Acute myocardial infarction in the elderly. Neth Heart J 23(10): 475- 476.

3. Dégano IR, Subirana I, Fusco D, Tavazzi L, Kirchberger I, et al. (2017) Percutaneous coronary intervention reduces mortality in myocardial infarction patients with comorbidities: Implications for elderly patients with diabetes or kidney disease. Int J Cardiol 249: 83-89.

4. Lin MJ, Lee J, Chen CY, Huang CC, Wu HP (2017) Chronic kidney disease and diabetes associated with long-term outcomes in patients receiving percutaneous coronary intervention. BMC Cardiovasc Disord 17: 242.

5. Feder SL, Schulman-Green D, Dodson JA, Geda M, Williams K, et al. (2016) Risk Stratification in Older Patients with Acute Myocardial Infarction: Physicians' Perspectives. J Aging Health 28(3): 387-402.

6. Fan Y, Jiang M, Gong D, Man C, Chen Y (2018) Cardiac troponin for predicting all-cause mortality in patients with acute ischemic stroke: a meta-analysis. Biosci Rep 38(2): BSR20171178.

7. Bassan F, Bassan R, Esporcatte R, Santos B, Tura B (2016) Very LongTerm Prognostic Role of Admission BNP in Non-ST Segment Elevation Acute Coronary Syndrome. Arq Bras Cardiol 106(3): 218-225.

8. Yoshinaga R, Doi Y, Ayukawa K, Ishikawa S (2017) High-sensitivity C reactive protein as a predictor of in hospital mortality in patients with cardiovascular disease at an emergency department: a retrospective cohort study. BMJ Open 7(10): e015112.

9. Thygesen Kristian, Alpert Joseph S, Jaffe Allan S, Simoons Maarten L, Chaitman Bernard R, et al. (2012) Third Universal Definition of Myocardial Infarction. Circulation 126(16): 2020-2035.

10. Tsai W, Chien DK, Huang CH, Shih SC, Chang WH (2017) Multiple Cardiac Biomarkers Used in Clinical Guideline for Elderly Patients with Acute Coronary Syndrome. Int J Gerontol 11(2): 104-108.

11. Eggers KM, Lindahl B (2017) Prognostic Biomarkers in Acute Coronary Syndromes: Risk Stratification Beyond Cardiac Troponins. Curr Cardiol Rep 19(4): 29.

12. Skau E, Henriksen E, Wagner P, Hedberg P, Siegbahn A, et al. (2017) GDF-15 and TRAIL-R2 are powerful predictors of long-term mortality in patients with acute myocardial infarction. Eur J Prev Cardiol 24(15): 1576-1583.

13. Chacko S, Haseeb S, Glover BM, Wallbridge D, Harper A (2017) The role of biomarkers in the diagnosis and risk stratification of acute coronary syndrome. Future Sci OA 4(1). 
14. Schellings DA, Adiyaman A, Dambrink J-HE, Gosselink AM, Kedhi E, et al. (2016) Predictive valueof NT-pro BNP for 30-day mortality in patients with non-ST-elevation acute coronary syndromes: a comparison with the GRACE and TIMI risk scores. Vasc Health Risk Manag 12: 471-476.

15. Kavsak PA, Neumann JT, Cullen L, Than M, Shortt C, et al. (2018) Clinical chemistry score versus high-sensitivity cardiac troponin I and $\mathrm{T}$ tests alone to identify patients at low or high risk for myocardial infarction or death at presentation to the emergency department. CMAJ Can Med Assoc J 190(33): E974-984.

16. Zhang S, Zhang HX, Lin RY, Zhang SM, Xu ZY (2016) Predictive role of NTpro BNP for adverse cardiac events in community-acquired pneumonia: a retrospective study Int J Clin Exp Med 9(7): 14411-14417.

17. Santarelli S, Russo V, Lalle I, De Berardinis B, Navarin S, et al. (2017) Usefulness of combining admission brain natriuretic peptide (BNP) plus hospital discharge bioelectrical impedance vector analysis (BIVA) in predicting 90 days cardiovascular mortality in patients with acute heart failure. Intern Emerg Med 12(4): 445-451.

18. Cheng H, Fan WZ, Wang SC, Liu ZH, Zang HL, et al. (2015) N-termina pro-brain natriuretic peptide and cardiac troponin I for the prognostic utility in elderly patients with severe sepsis or septic shock in intensive care unit: A retrospective study. J Crit Care 30(3): 654.e9-14.

19. Breining A, Negers A, Mora L, Moïsi L, Golmard JL, et al. (2018) Determinants of clinical presentation on outcomes in older patients with myocardial infarction. Geriatr Gerontol Int 18(12): 1591-1596.

20. Forcadell MJ, Vila-Córcoles A, De Diego C, Ochoa-Gondar O, Satué (2018) Incidence and mortality of myocardial infarction among Catalonian older adults with and without underlying risk conditions: The CAPAMIS study. Eur J Prev Cardiol 25(17): 1822-1830.

21. Krumholz HM, Normand SLT, Wang Y (2019) Twenty-Year Trends in Outcomes for Older Adults with Acute Myocardial Infarction in the United States Twenty-Year Trends in Outcomes of Acute Myocardial Infarction in Older US Adults Twenty-Year Trends in Outcomes of Acute Myocardial Infarction in Older US Adults. JAMA Netw Open 2(3): e191938-e191938.

ISSN: 2574-1241

DOI: 10.26717/BJSTR.2019.20.003462

Broslavskyte M. Biomed J Sci \& Tech Res

This work is licensed under Creative Commons Attribution 4.0 License

Submission Link: https://biomedres.us/submit-manuscript.php
22. Rubini Gimenez M, Twerenbold R, Reichlin T, Wildi K, Haaf P, et al (2014) Direct comparison of high-sensitivity-cardiac troponin I vs. T for the early diagnosis of acute myocardial infarction. Eur Heart J 35(34): 2303-2311.

23. Wang Y, Wang J, Wang X, Liu J, Jiang F, et al. (2017) Roles of ST2, IL-33 and BNP in predicting major adverse cardiovascular events in acute myocardial infarction after percutaneous coronary intervention. J Cell Mol Med 21(11): 2677-2684.

24. Parenica J, Kala P, Pavkova MG, Tomandl J, Spinar J, et al. (2016) Natriuretic peptides, nitrite/nitrate and superoxide dismutase have additional value on top of the GRACE score in prediction of one-year mortality and rehospitalisation for heart failure in STEMI patients Multiple biomarkers prospective cohort study. Int J Cardiol 211: 96-104.

25. Testa M, Rosso GL, Ferreri C, Feola M (2018) The Predictive Value of Plasma Brain Natriuretic Peptide and Galectin-3 in Elderly Patients Admitted for Heart Failure. Diseases 6(4).

26. Di Marca S, Rando A, Cataudella E, Pulvirenti A, Alaimo S, et al. (2018) B-type natriuretic peptide may predict prognosis in older adults admitted with a diagnosis other than heart failure. Nutr Metab Cardiovasc Dis NMCD 28(6): 636-642.

27. Rasmussen SH, Andersen-Ranberg K, Dahl JS, Nybo M, Jeune B, et al. (2019) Diagnosing heart failure in centenarians. J Geriatr Cardiol JGC 16(1): 1-11.

28. Plichart M, Orvoën G, Jourdain P, Quinquis L, Coste J, et al. (2017) Brain natriuretic peptide usefulness in very elderly dyspnoeic patients: the BED study. Eur J Heart Fail 19(4): 540-548.

29. Li Y, Zhong X, Cheng G, Zhao C, Zhang L, et al. (2017) Hs-CRP and allcause, cardiovascular, and cancer mortality risk: A meta-analysis. Atherosclerosis 259: 75-82.

30. Mani P, Puri R, Schwartz GG, Nissen SE, Shao M, et al. (2019) Association of Initial and Serial C-Reactive Protein Levels With Adverse Cardiovascular Events and Death After Acute Coronary Syndrome: A Secondary Analysis of the VISTA-16 Trial. JAMA Cardiol 4(4): 314-320.

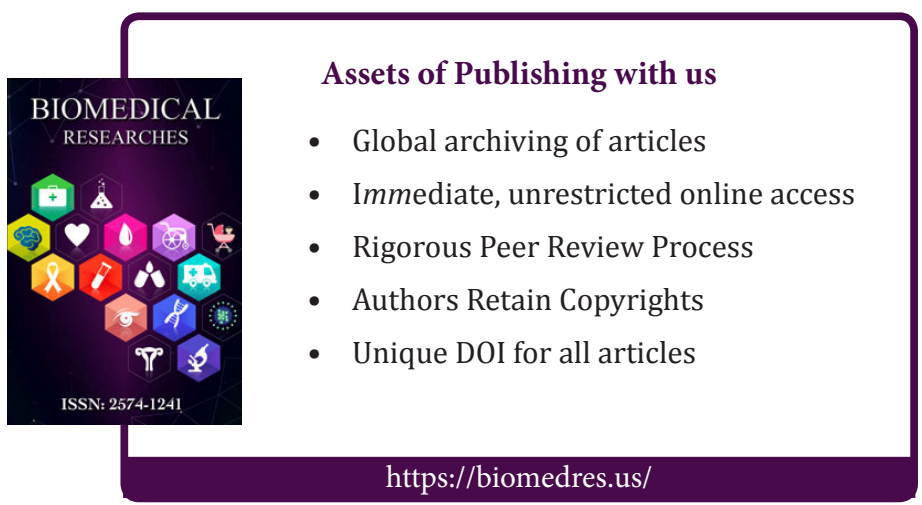

